VOL. $2(1970), 15-37$.

\title{
Generalisations of Turán's main theorems on lower bounds for sums of powers
}

\section{A. J. van der Poorten}

In his book, Eine neue Methode in der Analysis und deren Andwendungen, P. Turán proved a number of new theorems given lower bounds for sums of powers. Since it was only his intention to demonstrate a new type of result, his bounds are by no means best possible nor are his proofs easily susceptible of improvement. We generalise Turán's so-called Main Theorems to exponential sums with polynomial coefficients by a simple method involving only the evaluation and estimation of certain determinants. This approach gives in each case a result known to be asymptotically correct in the various exponents, and when specialised to the case of constant coefficients it provides in each case best-known results.

Our method moreover applies in more general circumstances and provided only that the determinants which arise can be conveniently estimated serves to provide lower bounds for other than exponential sums.

Let $\alpha_{1}, \alpha_{2}, \ldots, \alpha_{m}$ be complex numbers and $a_{1}, a_{2}, \ldots, a_{m}$ complex constants not all zero, and

Received 12 September 1969. The author wishes to acknowledge his indebtedness to $\mathrm{Dr}$ John Coates, who suggested to him the problems here studied, and to Professors Turán, Popken and Szekeres and Mr R. Tijdeman, who kindly pointed out errors and omissions in earlier drafts and versions of this work. 


$$
f(z)=\sum_{k=1}^{m} a_{k} \alpha_{k}^{z}
$$

Turán [8] considered lower bounds for the quantity

$$
\max _{n+1 \leq \mu \leq n+m} \frac{|f(\mu)|}{N(\mu, f)}
$$

where $N(\mu, f)$ is a suitable normalising factor so that the lower bound might be independent of either

(A) the $\alpha_{k}$-values

or (B) the $a_{k}$-coefficients.

We generalise Turán's theorems so that the $a_{k}$ become polynomial expressions in $z$.

Turán's Second Main Theorem [8; Satz IX, pp. 46-52] was to the effect that

$$
\max _{n+1 \leq \mu \leq n+m} \frac{\left|\sum_{k=1}^{m} a_{k} \alpha_{k}^{\mu}\right|}{\left|\alpha_{1}\right|^{\mu}} \geq\left(\frac{m}{24 e^{2}(n+2 m)}\right)^{m} \min _{z}\left|a_{1}+\ldots+a_{2}\right|
$$

where $\left|\alpha_{1}\right| \geq\left|\alpha_{2}\right| \geq \ldots \geq\left|\alpha_{m}\right|$. The quantity $Q_{1}=\left(\frac{m}{24 e^{2}(n+2 m)}\right)^{m}$ was improved by Vera T. Sós and P. Turán [7] who replaced it by $\left(\frac{m}{A(m+n)}\right)^{m}$ with $1.321<A<2 e^{1+4 / 3}(<24)$. Subsequently S. Uchiyama [9] improved a lower bound $1.473<A$ found by $E$. Makai [3] and showed that $e<A<8 e$, observing that the new upper bound was contained in the Sós and Turán paper [7]. E. Makai [4] then showed that $2 e / \log 2 \leq A$ and that we could take

$$
Q_{1}=\left(\frac{m}{A(m+n)}\right)^{m-1}
$$

giving the right order of magnitude in $n$. More recently Makai [6] constructed an example to show that in any event $4 e \leq A$.

We prove a more general result.

THEOREM 1. Let $\alpha_{1}, \alpha_{2}, \ldots, \alpha_{m}$ be complex numbers so arranged that 
$\left|\alpha_{1}\right| \geq\left|\alpha_{j}\right|, \quad 1 \leq j \leq m$, and $\left|\alpha_{1}-\alpha_{j}\right| \leq\left|\alpha_{1}-\alpha_{k}\right|$ for $1 \leq j \leq k \leq m$.

Denote by $E$ a function of the form

$$
F(z)=\sum_{k=1}^{m} p_{k}(z) \alpha_{k}^{z}=\sum_{k=1}^{m} \sum_{s=1}^{\rho(k)} a_{k s} z^{s-1} \alpha_{k}^{z}
$$

where $\rho(1), \ldots, \rho(m)$ are non-negative integers with sum $\sigma$, and $a_{k s}$ $1 \leq k \leq m, 1 \leq s \leq \rho(k)$, complex constants not all zero. Further write

$$
E_{l}(z)=\sum_{k=1}^{l} p_{k}(z) \alpha_{k}^{z}, \quad 1 \leq \ell \leq m \text {. }
$$

Then if $n, r, u$ are integers such that $n \geq-1,1 \leq r<\sigma$ and $u \leq n$,

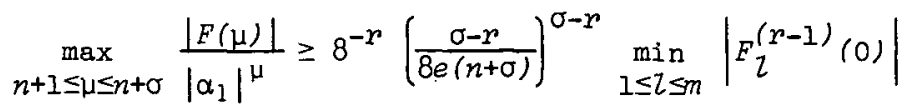

and

$$
\max _{n+1 \leq \mu \leq n+\sigma} \frac{|E(\mu)|}{\left|\alpha_{1}\right|^{\mu}} \geq \frac{1}{8}\left(\frac{\sigma-1}{8 e(n-\mu+\sigma)}\right)^{\sigma-1} \min _{1 \leq l \leq m}\left|F_{l}(u)\right| .
$$

COROLLARY. If $a_{1}, a_{2}, \ldots, a_{m}$ denote complex constants, then in particuzar

$$
\max _{n+1 \leq \mu \leq n+m} \frac{\left|\sum_{k=1}^{m} a_{k} \alpha_{k}^{\mu}\right|}{\left|\alpha_{1}\right|^{\mu}} \geq \frac{1}{8}\left(\frac{m-1}{8 e^{(m+n)}}\right)^{m-1} \min _{1 \leq l \leq m}\left|a_{1}+\ldots+a_{l}\right| .
$$

Apart from its greater generality our result is non-trivial in circumstances where Turán's result collapses. In the particular case of constant coefficients we do obtain the best-known result, of Makai [5].

Further Turán had shown (First Main Theorem) [8; Satz VII, pp. 38-41] that

$$
\max _{n+1 \leq \mu \leq n+m} \frac{\left|\sum_{k=1}^{m} a_{k} \alpha_{k}^{\mu}\right|}{\left|\alpha_{m}\right|^{\mu}} \geq\left(\frac{m}{2 e(n+m)}\right)^{m}\left|a_{1}+a_{2}+\ldots+a_{m}\right|
$$


where $\left|\alpha_{m}\right| \leq\left|\alpha_{j}\right|, I \leq j \leq m$. This result was improved by 1. Dancs [1] who replaced the quantity

$$
Q_{2}=\left(\frac{m}{2 e(n+m)}\right)^{m} \text { by } \frac{1}{2 e}\left(\frac{m}{2 e(n+m)}\right)^{m-1}
$$

which gave the correct order in $n$. Subsequently E. Makai [4] used a combinatorial argument to prove an exact result, showing the best possible value for $Q_{2}$ to be

$$
\left\{\sum_{z=0}^{m-1} 2^{2}\left(\begin{array}{c}
n+2 \\
z
\end{array}\right)\right\}^{-1}
$$

The corollary to our Theorem 2 is this best possible result.

-.THEOREM .2. Let $\alpha_{1}, \alpha_{2}, \ldots, \alpha_{m}$ be complex numbers so arranged that $\left|\alpha_{m}\right| \leq\left|\alpha_{j}\right|, \quad 1 \leq j \leq m$. Denote by $F$ a function of the form

$$
F(z)=\sum_{k=1}^{m} p_{k}(z) \alpha_{k}^{z}=\sum_{k=1}^{m} \sum_{s=1}^{\rho(k)} a_{k s^{z}} z^{s-1} \alpha_{k}^{z},
$$

where $\rho(1), \ldots, \rho(m)$ are non-negative integers with sum $\sigma$, and $a_{k s}$, $1 \leq k \leq m, 1 \leq s \leq \rho(k)$, complex constants not all zero. Then if $n, u$ are integers such that $n \geq-1, u \leq n$,

$$
\max _{n+1 \leq \mu \leq n+\sigma} \frac{|F(\mu)|}{\left|\alpha_{m}\right|^{\mu}} \geq \sum_{z=1}^{\sigma} 2^{z-1}\left(\begin{array}{c}
n-u+z-1 \\
z-1
\end{array}\right)^{-1} F(u),
$$

where we note that

$$
\sum_{z=1}^{\sigma}\left(\begin{array}{c}
n-u+z-1 \\
z-1
\end{array}\right) 2^{z-1} \leq\left(\begin{array}{c}
n-u+\sigma \\
\sigma-1
\end{array}\right) 2^{\sigma-1} \leq\left(\frac{2 e(n-u+\sigma)}{\sigma-1}\right)^{\sigma-1} .
$$

COROLLARY. If $a_{1}, a_{2}, \ldots, a_{m}$ denote complex constants, then in particular

$$
\max _{n+1 \leq \mu \leq n+m} \frac{\left|\sum_{k=1}^{m} a_{k} \alpha_{k}^{\mu}\right|}{\left|\alpha_{m}\right|^{\mu}} \geq \sum_{z=1}^{m} 2^{z-1}\left(\begin{array}{c}
n+z-1 \\
z-1
\end{array}\right)^{-1}\left|a_{1}+a_{2}+\ldots+a_{m}\right| .
$$

This is a best possible result in the sense that the right hand side cannot be replaced by a greater quantity for any $n$ or $m$. It seems 
likely that our general result similarly is exact. Indeed all bounds in our proof are exact, and in any event an argument we present at the end of this introduction shows that the general result is in a sense a special case of the Corollary.

Turán's Main Theorems are type-A results in that the lower bound is independent of the $\alpha_{k}$-values. Our method also easily provides a type- $B$ result where the lower bound is independent of the $a_{k s}$-coefficients.

Here Dancs and Turán [2] had shown that

$$
\max _{n+1 \leq \mu \leq n+m} \frac{\left|\sum_{k=1}^{m} \sum_{s=1}^{\rho} a_{k s} \mu^{s-1} \alpha_{k}^{\mu}\right|}{\sum_{k=1}^{m} \sum_{s=1}^{\rho}\left|a_{k s}\right| \mu^{s-1}\left|\alpha_{k}\right|^{\mu}} \geq(n+m \rho)^{-2 \rho^{2}}\left(\frac{\delta}{8 A}\right)^{2 m \rho+\frac{1}{2} p(\rho-1)},
$$

where

$$
\frac{\min _{1 \leq h \neq k \leq m}\left|\alpha_{h}-\alpha_{k}\right|}{\min _{1 \leq k \leq m}\left|\alpha_{k}\right|} \geq \delta \quad(\leq 1), \frac{\max _{1 \leq k \leq m}\left|\alpha_{k}\right|}{\min _{1 \leq k \leq m}\left|\alpha_{k}\right|} \leq A \quad(\geq 1)
$$

Whilst Coates [0] had proven

$$
\frac{\max _{0 \leq \mu \leq m \rho-1}\left|\sum_{k=1}^{m} \sum_{s=1}^{\rho} a_{k s} \mu^{s-1} \alpha_{k}^{\mu}\right|}{\max _{1 \leq k \leq m}\left|a_{k \rho}\right|} \geq \frac{(\rho-1) ! \delta^{(m-1) \rho}}{(6 A)^{p-1}} \times \min \left(1, A^{\prime m \rho-1}\right),
$$

where in addition $A^{\prime}=\max _{1 \leq k \leq m}\left|\alpha_{k}\right|$.

Our result generalises that of Coates and improves that of Dancs and Turán giving the correct exponents. In the particular case when the coefficients are constant our result is as good as that of Turán's book [8; Satz XI, pp. 53-56]. We prove

THEOREM 3. Let $\alpha_{1}, \alpha_{2}, \ldots, \alpha_{m}$ be distinct complex numbers, and denote by $F$ a function of the form

$$
F(z)=\sum_{k=1}^{m} p_{k}(z) \alpha_{k}^{z}=\sum_{k=1}^{m} \sum_{s=1}^{\rho(k)} a_{k s} z^{s-1} \alpha_{k}^{z},
$$

where $\rho(1), \ldots, \rho(m)$ are non-negative integers with sum $\sigma$, and $a_{k s}$, 
$I \leq k \leq m, I \leq s \leq \rho(k)$, complex constants not all zero. Further let

$$
\begin{aligned}
\delta_{h} & =\min _{1 \leq j \neq h \leq m}\left|\alpha_{h}-\alpha_{j}\right| ; A=\max _{1 \leq j \leq m}\left|\alpha_{j}\right|, \\
(1 \geq) \delta & =\min _{1 \leq h \leq m} \frac{\delta_{h}}{\alpha_{h}} ; \max _{1 \leq k \leq m} \rho(k)=\rho .
\end{aligned}
$$

Then if $n$ is an integer $\geq-1$, for each $h t, 1 \leq h \leq m$, $1 \leq t \leq \rho(h)$,

$$
\max _{n+1 \leq \mu \leq n+\sigma} \frac{|F(\mu)|}{\left|\alpha_{h t}\right|} \geq \frac{\left|\alpha_{h}\right|^{n+t} \delta_{h}^{\sigma-\rho(h)}\left\{\min \left(\left|\alpha_{h}\right|, \delta_{h}\right)\right\}^{\rho(h)-t}}{(n+\sigma)^{\rho(h)-t}(1+A)^{\sigma-1}} .
$$

COROLLARY 1.

$$
\max _{n+1 \leq \mu \leq n+\sigma} \frac{\left|\sum_{k=1}^{m} \sum_{s=1}^{\rho(k)} a_{k s} \mu^{s-1} \alpha_{k}^{\mu}\right|}{\sum_{k=1}^{m} \sum_{s=1}^{\rho(k)}\left|a_{k s}\right| \mu^{s-1}\left|\alpha_{k}\right|^{\mu}} \geq \frac{\delta^{\sigma-1}}{\sigma(n+\sigma)^{\rho-1}(1+A)^{\sigma-1}} .
$$

COROLLARY 2. In particular if $a_{1}, a_{2}, \ldots, a_{m}$ are complex constants

$$
\max _{n+1 \leq \mu \leq n+m} \frac{\left|\sum_{k=1}^{m} a_{k} \alpha_{k}^{\mu}\right|}{\left|a_{h}\right|} \geq \frac{\left|\alpha_{h}\right| n+1 \delta_{h}^{m-1}}{(1+A)^{m-1}}
$$

whence if $\min _{1 \leq j \leq m}\left|\alpha_{j}\right| \geq 1$,

$$
\max _{n+1 \leq \mu \leq n+m} \frac{\left|\sum_{k=1}^{m} a_{k} \alpha_{k}^{\mu}\right|}{\sum_{k=1}^{m}\left|a_{k}\right|\left|\alpha_{k}\right|^{\mu}} \geq \frac{\delta^{m-1}}{m(1+A)^{m-1}}
$$

Finally we illustrate how the expressions we study arise naturally. It is not aifficult to show that, given numbers $b_{0}, b_{1}, \ldots, b_{\sigma}$, the the solution to the difference equations

$$
b_{0} c_{n+\sigma}+b_{1} c_{n+\sigma-1}+b_{2} c_{n+\sigma-2}+\ldots+b_{\sigma} c_{n}=0, n=0,1,2, \ldots,
$$


is of the form

$$
c_{n}=p_{1}(n) \alpha_{1}^{n}+p_{2}(n) \alpha_{2}^{n}+\ldots+p_{m}(n) \alpha_{m}^{n}, n=0,1,2, \ldots,
$$

where

$$
b_{0} z^{\sigma}+b_{1} z^{\sigma-1}+\ldots+b_{\sigma}=b_{0}\left(z-\alpha_{1}\right)^{\rho(1)}\left(z-\alpha_{2}\right)^{\rho(2)} \ldots\left(z-\alpha_{m}\right)^{\rho(m)},
$$

and $p_{1}(t), \ldots, p_{m}(t)$ are polynomials of degree respectively at most $\rho(1)-1, \ldots, \rho(m)-1$.

It is of course evident from this description that the functions we study only represent a confluent case of those originally studied by Turán.

LEMMA 1. Let $F$ be a function of the form

$$
F(z)=\sum_{k=1}^{n} b_{k} g_{k}(z), \quad b_{1}, \ldots, b_{n} \text { complex constants, }
$$

where $g_{1}, \ldots, g_{n}$ are functions analytic on some domain $G$ of the complex plane.

Further let $z_{1}, z_{2}, \ldots, z_{n}$ be points of $G$; let $s_{1}, s_{2}, \ldots, s_{n}$ be non-negative integers; and let $H\left(y_{1}, \ldots, y_{l}\right)$ be a form linear in $y_{1}, \ldots, y_{2} \quad(1 \leq z \leq n)$.

Finally denote by $\Delta_{i j}(1 \leq i, j \leq n)$ the cofactor of $g_{j}{ }^{\left(s_{i}\right)}\left(z_{i}\right)$ in the determinant

$$
\Delta=\left|g_{j}{ }^{\left(s_{i}\right)}\left(z_{i}\right)\right|_{1 \leq j, i \leq n} .
$$

Then there is an integer $\mu$ such that $1 \leq \mu \leq n$ and

$$
\left|F^{\left(s_{\mu}\right)}\left(z_{\mu}\right)\right| \geq \frac{\left|H\left(b_{j(1)}, \ldots, b_{j(l)}\right)\right||\Delta|}{\sum_{i=1}^{n}\left|H\left(\Delta_{i j(1)}, \ldots, \Delta_{i j(1)}\right)\right|}, 1 \leq j(1) \leq \ldots \leq j(z) \leq n .
$$

Proof. Appropriately differentiating at $z_{1}, z_{2}, \ldots, z_{n}$ we obtain 
a system of $n$ linear equations in $b_{1}, \ldots, b_{n}$,

$$
\sum_{k=1}^{n} b_{k} g_{k}^{\left(s_{i}\right)}\left(z_{i}\right)=F^{\left(s_{i}\right)}\left(z_{i}\right), \quad 1 \leq i \leq n \text {, }
$$

which we may solve by Cramer's rule to obtain for $1 \leq k \leq n$

$$
b_{k} \Delta=\sum_{i=1}^{n} \Delta_{i k} F^{\left(s_{i}\right)}\left(z_{i}\right)
$$

Thus

$$
H\left(b_{j(1)}, \ldots, b_{j(l)}\right) \Delta=\sum_{i=1}^{n} H\left(\Delta_{i j(1)}, \ldots, \Delta_{i j(l)}\right) F^{\left(s_{i}\right)}\left(z_{i}\right)
$$

whence

$\left|H\left(b_{j(1)}, \ldots, b_{j(z)}\right)\right||\Delta| \leq \sum_{i=1}^{n}\left|H\left(\Delta_{i j(1)}, \ldots, \Delta_{i j(l)}\right)\right| \times \max _{1 \leq \mu \leq n}\left|F^{\left(s_{\mu}\right)}\left(z_{\mu}\right)\right|$, and the assertion follows. We note that the result remains meaningful though trivial even if the denominator on the right-hand side of the result should vanish, provided we then interpret the lower bound to be zero (this is the natural interpretation, in our examples, by continuity).

The Lemma reduces the problem of determining lower bounds like those of Turán to that of finding upper bounds for quotients of the form

$$
\sum_{i=1}^{n}\left|H\left(\Delta_{i j(1)}, \ldots, \Delta_{i j(2)}\right)\right| /|\Delta| \text {. }
$$

One useful technique runs as follows: writing

$$
q_{i}=H\left(\Delta_{i j(1)}, \ldots, \Delta_{i j(2)}\right) / \Delta, \quad 1 \leq i \leq n,
$$

we find a polynomial $Q$ such that

$$
Q(z)=q_{1}+q_{2} z+q_{3} z^{2}+\ldots+q_{n} z^{n} .
$$

LEMMA 2. If $Q(z)$ is given as a swo of terms of the form

$$
\delta\left(z-\beta_{1}\right)\left(z-\beta_{2}\right) \ldots\left(z-\beta_{p}\right)
$$

say 


$$
Q(z)=\sum_{j} \delta_{j}\left(z-\beta_{j 1}\right) \ldots\left(z-\beta_{j r}(j)\right.
$$

then

$$
\sum_{i=1}^{n}\left|q_{i}\right| \leq \sum_{j}\left|\delta_{j}\right|\left(I+\left|\beta_{j l}\right|\right) \ldots\left(1+\left|\beta_{j r}(j)\right|\right) .
$$

Proof. $\sum_{i=1}^{n}\left|q_{i}\right|$ is the sum of the absolute values of the coefficients of $Q$. But

$$
|\delta|\left(1+\left|\beta_{1}\right|\right) \ldots\left(1+\left|\beta_{p}\right|\right)
$$

is obviously as large as the sum of the absolute values of the coefficients of the polynomial

$$
\delta\left(z-\beta_{1}\right) \quad \ldots\left(z-\beta_{r}\right)
$$

whence the given inequality follows from the triangle inequality for the absolute value of complex numbers.

Henceforth $\rho(1), \ldots, \rho(m)$ denote non-negative integers with sum

$$
\sum_{k=1}^{m} \rho(k)=\sigma
$$

and $a_{k s}, I \leq k \leq m, I \leq s \leq \rho(k)$, denote $\sigma$ complex numbers not all zero. Further $p_{1}, \ldots, p_{m}$ denote the polynomials

$$
p_{k}(z)=\sum_{s=1}^{\rho(k)} a_{k s} z^{s-1}
$$

of respective degree at most $\rho(1)-1, \ldots, \rho(m)-1$ (and the convention that a polynomial of degree $<0$ is identically zero).

We will consider functions $F$ of the form

$$
F(z)=\sum_{k=1}^{m} p_{k}(z) f_{k}(z)=\sum_{k=1}^{m} \sum_{s=1}^{\rho(k)} a_{k s^{s}} z^{s-1} f_{k}(z)
$$

so that we will be applying the main lemma (Lemma 1) to $\sigma$ functions

$$
z^{s-1} f_{k}(z), \quad 1 \leq k \leq m, \quad 1 \leq s \leq \rho(k),
$$


and evaluating cofactors of $\sigma \times \sigma$ determinants of the form

$$
\Delta=\left|z_{i}^{t-1} f_{h}^{\left(s_{i}\right)}\left(z_{i}\right)\right|_{h t, i}, 1 \leq i \leq \sigma ; 1 \leq t \leq \rho(h), \quad 1 \leq h \leq m .
$$

Here $h, t$ index rows and $i$ indexes columns; the above $h t, i$ index indicating that we are displaying the $h t, i$ element of the determinant. We always denote the cofactor, i.e. the determinant of the minor together with the appropriate sign, of the $h t, i$ element $\Delta_{i, h t}$.

On occasion it will be appropriate to note explicitly that, say, the ht row is strictly the $\rho(1)+\ldots+\rho(h-1)+t$ row; we then write $\rho(1)+\ldots+\rho(h-1)+t=\sigma(h t)$ for convenience.

Turán's Main Theorems concern lower bounds for sums of powers of complex numbers. We shall more generally consider such sums with polynomial coefficients, and thus define

$$
f_{1}(z)=e^{\omega_{1} z}=\alpha_{1}^{z}, \ldots, f_{m}(z)=e^{\omega_{m}^{z}}=\alpha_{m}^{z}
$$

where $\omega_{1}, \omega_{2}, \ldots, \omega_{m}$ are arbitrary complex numbers (in the extended sense, so that $\alpha_{h}$ may be zero) and $\alpha_{1}^{z}, \alpha_{2}^{z}, \ldots, \alpha_{m}^{z}$ are well defined if $z$ takes only rational integer values; we use the $\alpha^{\prime}$ s only in that context.

Since the essence of our method is the evaluation or estimation of certain determinants and their cofactors, we describe precisely those cases for which this may be conveniently done.

To begin with we will find the lower bounds for

$$
\max _{\mu=n+1, \ldots, n+\sigma}\left|F(\mu)=\sum_{k=1}^{m} p_{k}(\mu) \alpha_{k}^{\mu}\right| \text {. }
$$

By the main lemma we must then consider the determinant

$$
\Delta=\left|(n+i)^{t-1} \alpha_{h}^{n+i}\right|_{h t, i}, 1 \leq h \leq m, 1 \leq t \leq \rho(h) ; 1 \leq i \leq \sigma,
$$

and various linear forms in its cofactors. Different such linear forms will provide us with lower bounds appropriate in different circumstances.

Our technique for evaluating $\Delta$ and expressions in its cofactors runs 
as

follows: we introduce formal quantities

$$
\alpha_{h t}, \quad 1 \leq h \leq m ; 1 \leq t \leq \rho(h)
$$

and the Vandermonde determinant

$$
D=\left|\alpha_{h t}^{n+i}\right|_{h t, i}
$$

$D$ is of course easily evaluated, and we obtain

$$
D=\prod_{k=1}^{m} \prod_{s=1}^{p(k)}\left\{\alpha_{k s}^{n+1} \prod_{j r<k s}\left(\alpha_{k s}-\alpha_{j r}\right)\right\}
$$

(where of course $j r<k s$ means $\sigma(j r)<\sigma(k s)$ i.e. either $j<k$ or $j=k$ and $r<s)$, whence more conveniently

$$
D=\prod_{k=1}^{m} \prod_{s=1}^{\rho(k)}\left\{\alpha_{k s}^{n+1}\left(\prod_{l=1}^{s-1}\left(\alpha_{k s}-\alpha_{k l}\right)\right) \prod_{j=1}^{k-1} \prod_{r=1}^{\rho(k)}\left(\alpha_{k s}-\alpha_{j r}\right)\right\}
$$

But it is easily seen that exactly

$$
\begin{aligned}
\Delta & =\underset{\substack{\alpha_{k s} \rightarrow \alpha_{k} \\
1 \leq k \leq m ; 1 \leq s \leq \rho(k)}}{\lim }\left(\left\{\prod_{k=1}^{m} \prod_{s=1}^{\rho(k)}\left(\alpha_{k s} \frac{\partial}{\partial \alpha_{k s}}\right)^{s-1}\right\}\right) \\
& =\prod_{k=1}^{m} \prod_{s=1}^{\rho(k)}\left\{\alpha_{k}^{n+s}(s-1) ! \prod_{j=1}^{k-1}\left(\alpha_{k}-\alpha_{j}\right)^{\rho(j)}\right\} .
\end{aligned}
$$

The reader may be able to see that the differentiation gives the above result; to describe the situation more clearly we however explicitly indicate how and why we obtain a tidy result in this and slightly more general circumstances.

LEMMA 3. Denote by $P$ the product

$$
P=\prod_{k=1}^{m} \prod_{s=1}^{\rho(k)} \prod_{l=1}^{s-1}\left(\alpha_{k s}-\alpha_{k l}\right)
$$

and let $R_{1}, R_{2}$ be functions in the $\alpha_{h t}, 1 \leq h \leq m ; 1 \leq t \leq \rho(h)$, which are divisible by $P$, i.e. so that

$$
\lim _{\alpha_{k s}+\alpha_{k}} \quad R_{i / P} \quad(i=1,2)
$$

$1 \leq k \leq m ; 1 \leq 8 \leq \rho(k)$ 
exists (in effect every factor in $P$ occurs in an appropriate factorisation of $R$ ). Then

$$
\lim _{\substack{\alpha_{k s} \rightarrow \alpha_{k} \\ \text { all .ks }}}\left(\frac{\left\{\prod_{k=1}^{m} \prod_{s=1}^{p(k)}\left(\alpha_{k s} \frac{\partial}{\partial \alpha_{k s}}\right)^{s-1}\right\} R_{1}}{\left.\left\{\prod_{s=1}^{p(k)}\left(\alpha_{k s} \frac{\partial}{\partial \alpha_{k s}}\right)^{s-1}\right\}_{2}\right)}=\underset{\substack{\alpha_{k s} \rightarrow \alpha_{k} \\ \text { all } k s}}{\lim _{2 / P}}\left(\frac{R_{1 / P}}{R_{2 / P}}\right) .\right.
$$

Proof. Write $R=P Q$. Then $\lim _{\substack{\alpha_{k s} \rightarrow \alpha_{k} \\ \text { all } k s}}\left(\left\{\prod_{k=1}^{m} \prod_{s=1}^{\rho(k)}\left(\alpha_{k s} \frac{\partial}{\partial \alpha_{k s}}\right)^{s-1}\right\} P Q\right)=\underset{\substack{\alpha_{k s} \rightarrow \alpha_{k} \\ \text { all } k s}}{\lim }\left(Q\left\{\prod_{k=1}^{m} \prod_{s=1}^{\rho(k)}\left(\alpha_{k s} \frac{\partial}{\partial \alpha_{k s}}\right)^{s-1}\right\} P\right.$

+ terms which vanish when limits are taken)$$
=\left(\begin{array}{ll}
\lim _{\alpha_{k s} \rightarrow \alpha_{k}} & Q
\end{array}\right) \cdot \prod_{k=1}^{m} \prod_{s=1}^{\rho(k)}(s-1) !
$$

all ks

and the result of the lema follows immediately.

With the aid of Lemma 3 it is now easy to give explicit expressions for such combinations of the cofactors of $\Delta$ as are, in the sense of the lemma, divisible by $P$. In other cases we unfortunately obtain an annoyingly untidy expression.

We denote by $D_{i, h t}$ and respectively $\Delta_{i, h t}$ the cofactor of $\alpha_{h t}^{n+i}$ and respectively $(n+i)^{t-1} \alpha_{h}^{n+i}$ in $D$ and respectively $\Delta$. In just the same way as we derived $\Delta$ from $D$ it is clear that

$$
\Delta_{i, h t}=\lim _{\substack{\alpha_{k s} \rightarrow \alpha_{k} \\ \text { all ks }}}\left(\left\{\prod_{\substack{k=1 \\ k s \neq h t}}^{m} \prod_{\substack{s=1 \\(k)}}^{\rho(k)}\left(\alpha_{k s} \frac{\partial}{\partial \alpha_{k s}}\right)^{s-1}\right\} D_{i, h t}\right) \text {. }
$$

But the $D_{i, h t}$ are determined by the equations

$$
\frac{D}{\alpha_{h t}^{n+1}} \delta_{k s, h t}=\sum_{i=1}^{\sigma} \alpha_{k s}^{i-1} D_{i, h t}, \quad 1 \leq k \leq m ; 1 \leq s \leq \rho(k)
$$

(where $\delta_{k s, h t}$ is the well-known Kronecker $\delta$ ), which assert that $D_{i, h t}$ 
is exactly the coefficient of $z^{i-1}$ in the polynomial

$$
\frac{D}{\alpha_{h t}^{n+1}} \prod_{\substack{k=1 \\ k s \neq h t}}^{m} \prod_{\substack{s=1 \\(k)}}\left(\frac{z-\alpha_{k s}}{\alpha_{h t}-\alpha_{k s}}\right) \text {. }
$$

Unfortunately this expression is not, in the sense of Lemma 3 , divisible by the product $P$ so that we must obtain an unpleasant expression for the cofactors $\Delta_{i, h t}$. We return to that expression later; for the moment we list various linear forms in the $\Delta_{i, h t}$ for which we can obtain a relatively tidy exact expression.

Thus for any $\mu$ we have by the above expressions

$$
\alpha_{h}^{u} \sum_{t=1}^{\rho(h)} u^{t-1} \Delta_{i, h t}=\lim _{\substack{\alpha_{k s} \rightarrow \alpha_{k} \\ \text { all } k s}}\left(\left\{\prod_{k=1}^{m} \prod_{s=1}^{\rho(k)}\left(\alpha_{k s} \frac{\partial}{\partial \alpha_{k s}}\right)^{s-1}\right\} \cdot \sum_{t=1}^{\rho(h)} \alpha_{h t}^{u} D_{i, h t}\right)
$$

and by Lemma 3 and the above expressions

$$
=\text { the coefficient of } z^{i-1} \text { in the polynomial }
$$

$$
\lim _{\substack{\alpha_{k s} \rightarrow \alpha_{k} \\ \text { all ks }}} \Delta \sum_{t=1}^{\rho(h)} \frac{1}{\alpha_{h t}^{n+1-u}} \prod_{\substack{k=1 \\ k s \neq h t}}^{m} \prod_{\substack{s=1 \\ k t}}^{\rho(k)}\left(\frac{z-\alpha_{k s}}{\alpha_{h t}-\alpha_{k s}}\right) \text {. }
$$

Similarly for $r$ a positive integer and a fixed branch of the log function

$$
\begin{aligned}
& \sum_{t=1}^{\rho(h)} \frac{(r-1) !}{(r-t) !}\left(\log \alpha_{h}\right)^{r-t} \Delta_{i, h t} \\
& \quad=\lim _{\substack{\alpha_{k s} \rightarrow \alpha_{k} \\
\text { all ks }}}\left(\left\{\prod_{k=1}^{m} \prod_{s=1}^{\rho(k)}\left(\alpha_{k s} \frac{\partial}{\partial \alpha_{k s}}\right)^{s-1}\right\}^{\rho(h)} \sum_{t=1}^{\left.\left.\rho \log \alpha_{h t}\right)^{r-1} D_{i, h t}\right),}\right.
\end{aligned}
$$

which by Lemma 3 becomes 


$$
=\text { the coefficient of } z^{i-1} \text { in the polynomial }
$$

$$
\lim _{\substack{\alpha_{k s} \rightarrow \alpha_{k} \\ \text { all } k s}} \Delta \sum_{t=1}^{\rho(h)} \frac{\left(\log \alpha_{h t}\right)^{r-1}}{\alpha_{h t}^{n+1}} \prod_{\substack{k=1 \\ k s \neq h t}}^{m} \prod_{s=1}^{\rho(k)}\left(\frac{z-\alpha_{k s}}{\alpha_{h t}-\alpha_{k s}}\right) \text {. }
$$

We consider the following linear form in the $\Delta_{i, h t}$

$$
\sum_{h=t}^{l} \sum_{t=1}^{\rho(h)} \frac{(r-1) !}{(r-1) !}\left(\log \alpha_{h}\right)^{r-t} \frac{\Delta_{i, h t}}{\Delta}=q_{i, i}=q_{i} \quad(1 \leq r<\sigma)
$$

where $1 \leq l \leq m$; the corresponding linear form in the coefficients $a_{k s}$ is then

$$
\sum_{h=1}^{l} \sum_{t=1}^{\rho(h)} \frac{(r-1) !}{(r-1) !}\left(\log \alpha_{h}\right)^{r-t} a_{h t}=\left\{\left(\frac{d}{d z}\right)^{r-1} \sum_{h=1}^{l} p_{h}(z) \alpha_{h}^{z}\right\}_{z=0} .
$$

For convenience writing

$$
F_{2}(z)=\sum_{h=1}^{Z} p_{h}(z) \alpha_{h}^{z}=\sum_{h=1}^{l} \sum_{t=1}^{\rho(h)} a_{h t^{z}}{ }^{t-1} \alpha_{h}^{z}, \quad 1 \leq \imath \leq m,
$$

(so that $F_{m}(z)=F(z)$ ), the above linear form is $F_{l}^{(r-1)}(0)$.

We have observed above that $q_{i}$ is exactly the coefficient of $z^{i-1}$ in the polynomial

$$
Q_{Z}(z)=\lim _{\substack{\alpha_{k s} \rightarrow \alpha_{k} \\ \text { all ks }}} \sum_{h=1}^{l} \sum_{t=1}^{\rho(h)} \frac{\left(\log \alpha_{h t}\right)^{r-1}}{\alpha_{h t}^{n+1}} \prod_{\substack{k=1 \\ k s \neq h t}}^{m} \prod_{\substack{s=1 \\ k s}}^{\rho(k)}\left(\frac{z-\alpha_{k s}}{\alpha_{h t}-\alpha_{k s}}\right) .
$$

Neglecting the limit for a moment, $Q_{Z}(z)$ is exactly that polynomial of degree $\sigma-1$ such that

$$
\begin{array}{rlrl}
Q_{2}\left(\alpha_{h t}\right) & =\frac{\left(\log \alpha_{h t}\right)^{r-1}}{\alpha_{h t}^{n+1}} & 1 \leq h \leq \imath ; 1 \leq t \leq \rho(h) ; \\
& =0 & l+1 \leq h \leq m ; 1 \leq t \leq \rho(h) .
\end{array}
$$

Taking the quantities $\alpha_{h t}$ as formally distinct these conditions 
determine $Q_{\ell}$.

Suppose now that

$$
\max _{I \leq j \leq m}\left|\alpha_{j}\right|=1
$$

and rearrange $\alpha_{1}, \alpha_{2}, \ldots, \alpha_{m}$ such that $\alpha_{1}=1$ and

$$
0=\left|1-\alpha_{1}\right| \leq \ldots \leq\left|1-\alpha_{\eta}\right|<R<\left|1-\alpha_{\eta+1}\right| \leq \ldots \leq\left|1-\alpha_{m}\right|
$$

where $R$ and the integer $l, l \leq l \leq m$, are to be fixed later. We suppose that the distinct quantities $\alpha_{h t}$ lie arbitrarily close to the $\alpha_{h} \quad l \leq h \leq m$.

Still neglecting the limit in $Q_{\eta}(z)$ we write it as an interpolation series

(6) $Q_{2}(z)=b_{11}+b_{12}\left(z-\alpha_{11}\right)+b_{13}\left(z-\alpha_{11}\right)\left(z-\alpha_{12}\right)+\ldots+b_{m \rho(m)}\left(z-\alpha_{11}\right) \ldots\left(z-\alpha_{m, \rho}(m)-1\right)$. By the conditions (4) defining $Q_{l}$ we obtain the interpolation coeficients $b_{h t}$ as contour integrals

(7) $b_{h t}=\frac{1}{2 \pi i} \int_{C} \frac{(\log z)^{p-1} d z}{z^{n+1}\left(z-\alpha_{11}\right) \ldots\left(z-\alpha_{h t}\right)}, \quad 1 \leq h \leq m ; 1 \leq t \leq \rho(h)$, where the contour includes all of the points $\alpha_{1}, \ldots, \alpha_{2}$ but excludes the points $\alpha_{z+1}, \ldots, \alpha_{m}$ and the origin. The integrals remain well defined when the relevant limit is taken and indeed we can drop any implicit assumption that the $\alpha_{h}$ be distinct. For our contour we will take the circle $|1-z|=R$ assuming then that $R$ is chosen $<1$. We sketch the procedure we now follow. In order to determine a lower bound for

$$
\max _{\mu=n+1, \ldots, n+\sigma}\left|F(\mu)=\sum_{k=1}^{m} p_{k}(\mu) \alpha_{k}^{\mu}\right|
$$

we require by Lemma 1 to find an upper bound for the sum 


$$
\sum_{i=1}^{\sigma}\left|a_{i}\right|
$$

and by Lemma 2 we know that

$$
\sum_{h=1}^{m} \sum_{t=1}^{\rho(h)}\left|b_{h t}\right|\left(1+\left|\alpha_{11}\right|\right) \ldots\left(1+\left|\alpha_{h, t-1}\right|\right)
$$

is such an upper bound. To determine this bound we require the following lemma of Makai [5; pp. 180-181], an analogue of a well known theorem of Chebychev. We quote the lemma without proof.

LEMMA (Makai). If $0 \leq \delta \leq 1$, and $P(z)$ is a monic polynomial of degree $n$ then there exists a circle $|1-z|=R$ with $0<R<\delta$ such that everywhere on the circle

$$
|P(z)| \geq 2(\delta / 4)^{n} .
$$

We thus choose $\delta$ (we determine a specific optimal choice later) and thus $R$ and $Z$ such that

$$
\left|1-\alpha_{\eta}\right|<R<\delta \leq\left|1-\alpha_{2+1}\right| \text {. }
$$

Then everywhere on the contour $|1-z|=R$ we have

$$
\left|\frac{(\log z)^{r-1}}{z^{n+1}}\right| \leq \frac{\{-\log (1-\delta)\}^{r-1}}{(1-\delta)^{n+1}}<\frac{(\delta / 1-\delta)^{r-1}}{(1-\delta)^{n+r}}<\frac{\delta^{r-1}}{(1-\delta)^{n+r}}
$$

and by Makai's lenma on the contour

$$
\left|\left(z-\alpha_{11}\right) \ldots\left(z-\alpha_{m \rho(m)}\right)\right| \geq 2(\delta / 4)^{\sigma},
$$

whence a fortiori, certainly

$$
\left|\left(z-\alpha_{11}\right) \ldots\left(z-\alpha_{h t}\right)\right| \geq 2(\delta / 4)^{\sigma}, \quad 1 \leq h \leq m ; 1 \leq t \leq \rho(h) .
$$

Hence we see that

$$
\left|b_{h t}\right| \leq \frac{1}{2 \pi} \cdot 2 \pi \delta \cdot \frac{\delta^{r-1}}{(1-\delta)^{n+r}} \cdot \frac{2^{2 \sigma-1}}{\delta^{\sigma}} \leq \frac{2^{2 \sigma-1}}{\delta^{\sigma-r}(1-\delta)^{n+r}} .
$$

Recalling that $\left|\alpha_{h}\right| \leq 1 \quad(1 \leq h \leq m)$ we obtain that 


$$
\begin{aligned}
\sum_{i=1}^{\sigma}\left|a_{i}\right| & \left.\leq \sum_{h=1}^{m} \sum_{t=1}^{\rho(h)}\left|b_{h t}\right|\left(1+\left|\alpha_{11}\right|\right) \cdots\left(1+\left|\alpha_{h, t-1}\right|\right) \text { (where } \alpha_{h t} \equiv \alpha_{h}\right) \\
& \leq \sum_{i=1}^{\sigma} \frac{2^{2 \sigma-1}}{\delta^{\sigma-r}(1-\delta)^{n+r}} \cdot 2^{i-1} \\
& \leq 2^{3 \sigma-1} / \delta^{\sigma-r} \cdot(1-\delta)^{n+r} .
\end{aligned}
$$

We are still free to choose $\delta$ optimally (thus determining 2 ). It is easy to see that the best choice is

$$
\left.\delta=\frac{\sigma-r}{n+\sigma} \quad \text { (recalling } \quad 1 \leq r \leq \sigma\right)
$$

and then

$$
\begin{gathered}
(1-\delta)^{-(n+r)}=\left(1+\frac{\sigma-r}{n+r}\right)^{n+r}<e^{\sigma-r}, \\
\delta^{-(\sigma-r)}=\left(\frac{n+\sigma}{\sigma-r}\right)^{\sigma-r},
\end{gathered}
$$

whence collecting our results we have shown that

(8) $\min _{1 \leq l \leq m} \sum_{i=1}^{\sigma}\left|\sum_{h=1}^{l} \sum_{t=1}^{\rho(h)} \frac{(r-1) !}{(r-t) !}\left(\log \alpha_{h}\right)^{r-t} \frac{\Delta_{i, h t}}{\Delta}\right| \leq 8^{r}\left(\frac{8 e(n+\sigma)}{\sigma-r}\right)^{\sigma-r}$, provided only that $\alpha_{1}, \alpha_{2}, \ldots, \alpha_{m}$ are as in (5), and $r<\sigma$.

Taking $r=1$ and comparing the expressions (2) and (1) observing that the latter is simply the former with $r=1$ and $n$ replaced by $n-u$ we can read off the following result:

$$
\min _{1 \leq l \leq m} \sum_{i=1}^{\sigma}\left|\sum_{h=1}^{l} \rho \sum_{t=1}^{\rho(h)} \alpha_{h}^{u} u^{t-1} \frac{\Delta_{i, h t}}{\Delta}\right| \leq 8\left(\frac{8 e(n-u+\sigma)}{\sigma-1}\right)^{\sigma-1}
$$

when $\alpha_{1}, \alpha_{2}, \ldots, \alpha_{m}$ are as in (5) and $u<n+1$ (this condition is required to preserve our estimates which, perhaps implicitly, are based on the premiss that $n$, or in this case $n-u$, is large relative to the other constants).

We note that the relevant linear form to this last result is

$$
\sum_{h=1}^{l} \sum_{t=1}^{\rho(h)} \alpha_{h}^{u} u^{t-1} a_{h t}=\sum_{h=1}^{l} p_{h}(u) \alpha_{h}^{u}=F_{l}(u) \text {. }
$$


Finally the above upper bounds for the relevant linear forms and Lemma 1 immediately give the results quoted as Theorem 1 , for by the Lemma we immediately have that there is a $\mu$ such that $I \leq \mu \leq \sigma$ and

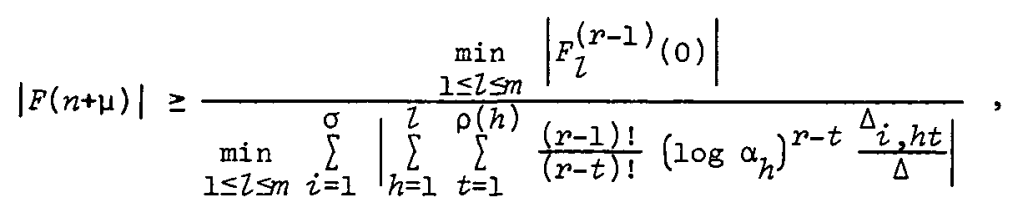

the numerator and denominator being respectively the same linear form in respectively the coefficients $a_{k s}$ and the cofactors $\Delta_{i, k s}$ by (3) whilst similarly by (10) we see that there is a $\mu$ such that $1 \leq \mu \leq \sigma$ and

$$
|F(n+\mu)| \geq \frac{\min _{1 \leq l \leq m}\left|F_{\imath}(u)\right|}{\min _{l \leq l \leq m} \sum_{i=1}^{\sigma}\left|\sum_{h=1}^{l} p(h) \sum_{t=1}^{u} \alpha_{h} u^{t-1} \frac{\Delta_{i, h t}}{\Delta}\right|} .
$$

Further we may, since $F(z)$ is homogeneous in the $\alpha_{h}^{z}$, make the relevant changes to the condition (5) that we make in our statement of the theorem. If in contrast to the condition (5) on the $\alpha_{h}$ we have

$$
\min _{1 \leq j \leq m}\left|\alpha_{j}\right|=1
$$

say $\alpha_{m}=1$, we obtain a quite different result. We consider the linear form

$$
\sum_{h=1}^{m} \sum_{t=1}^{\rho(h)} \alpha_{h}^{u} u^{t-1} \frac{\Delta_{i, h t}}{\Delta},
$$

which we have seen by $(2,3)$ is tantamount to considering

$$
\sum_{h=1}^{m} \sum_{t=1}^{\rho(h)} \frac{\Delta_{i, h t}}{\Delta}
$$

with $n$ replaced by $n-u$. We thus follow the same chain of argument as before to obtain 
(11) $\sum_{i=1}^{\sigma} \sum_{h=1}^{m}\left|\sum_{t=1}^{\rho(h)} \alpha_{h}^{u} u^{t-1} \frac{\Delta_{i, h t}}{\Delta}\right| \leq \sum_{h=1}^{m} \rho \sum_{t=1}^{\rho(h)}\left|b_{h t}\right|\left(1+\left|\alpha_{11}\right|\right) \ldots\left(1+\left|\alpha_{h, t-1}\right|\right)$, where

$$
b_{h t}=\frac{1}{2 \pi i} \int_{C} \frac{d z}{z^{n-u+1}\left(z-\alpha_{11}\right) \ldots\left(z-\alpha_{h t}\right)} \text {, }
$$

$\alpha_{h t}=\alpha_{h}, 1 \leq h \leq m ; 1 \leq t \leq \rho(h)$, and the contour is such as to contain $\alpha_{1}, \alpha_{2}, \ldots, \alpha_{m}$ but exclude the origin. We suppose that $u$ is an integer and $<n+1$. Then the only singularity outside the contour in the above integrands is a multiple pole of the origin, and to be explicit we may take as contour $C$ a small circle (radius < 1 ) about the origin in the negative direction. In order to evaluate the residue of the integrand as $z=0$ we note that on the contour always $|z|<\left|\alpha_{k s}\right|$ whence

$$
\left\{\left(z-\alpha_{11}\right) \ldots\left(z-\alpha_{h t}\right)\right\}^{-1}=\left\{(-1)^{\sigma(h t)} \alpha_{11} \ldots \alpha_{h t}\left(1-z / \alpha_{11}\right) \ldots\left(1-z / \alpha_{h t}\right)\right\}^{-1}
$$

(where $\sigma(h t)=\rho(1)+\ldots+\rho(h-1)+t)$

$$
=(-1)^{\sigma(h t)}\left(\alpha_{11} \ldots \alpha_{h t}\right)^{-1} \sum_{\mu=0}^{\infty} c_{\mu, h t^{z^{\mu}}}
$$

and

$$
c_{\mu, h t}=\sum^{\mu} \alpha_{11}^{-\lambda(11)} \alpha_{12}^{-\lambda(12)} \ldots \alpha_{h t}^{-\lambda(h t)}
$$

where the sum is taken over all non-negative integers $\lambda(1 I), \ldots, \lambda(h t)$ with sum $\mu$.

Since in any event $\left|\alpha_{k s}\right| \geq 1$, all $k s, c_{\mu}$ is maximal when $\alpha_{1}=\alpha_{2}=\ldots=\alpha_{m}$. Then $c_{\mu}$ is the coefficient of $z^{\mu}$ in the power series expansion of $(1-z)^{-\sigma(h t)}$ and it follows that

$$
c_{\mu, h t} \leq\left(\begin{array}{c}
\mu+\sigma(h t)-1 \\
\sigma(h t)-1
\end{array}\right)=\frac{(\mu+\sigma(h t)-1) !}{(\sigma(h t)-1) ! \mu !} .
$$

Finally applying the residue theorem to the integral (12) we obtain

$$
\left|b_{h t}\right|=\left|\left(\alpha_{11} \ldots \alpha_{h t}\right)^{-1}\right|\left|c_{n-\mu, h t}\right|
$$


whence

$$
\left|\alpha_{11} \ldots \alpha_{h t}\right| \quad\left|b_{h t}\right| \leq\left(\begin{array}{c}
n-u+\sigma(h t)-1 \\
\sigma(h t)-1
\end{array}\right)
$$

But then

$\left|b_{h t}\right|\left(1+\left|\alpha_{11}\right|\right) \ldots\left(1+\left|\alpha_{h, t-1}\right|\right)$

$=\left|\alpha_{11} \ldots \alpha_{h t}\right|\left|b_{h t}\right| \cdot\left|\alpha_{h t}^{-1}\right|\left(\left|\alpha_{11}^{-1}\right|+1\right) \ldots\left(\left|\alpha_{h, t-1}^{-1}\right|+1\right) \leq\left(\begin{array}{c}n-u-\sigma(h t)-1 \\ \sigma(h t)-1\end{array}\right) 2^{\sigma(h t)-1}$.

Thus by (11)

$$
\sum_{i=1}^{\sigma}\left|\sum_{h=1}^{m} \sum_{t=1}^{\rho(h)} \alpha_{h}^{u} u^{t-1} \frac{\Delta_{i, h t}}{\Delta}\right| \leq \sum_{r=1}^{\sigma}\left(\begin{array}{c}
n-u+r-1 \\
r-1
\end{array}\right) 2^{r-1} .
$$

By just those observations we made at the end of the proof of Theorem 1 , Theorem 2 now follows.

lurán's Main Theorems, which our Theorems 1 and 2 generalise, are A-type inequalities in the sense of our introduction; we now turn to a corresponding $B$-type inequality.

By Lemma 1 we only require an upper bound for $\sum_{i=1}^{\sigma}\left|\frac{\Delta_{i, h t}}{\Delta}\right|$ since

$$
\max _{1 \leq \mu \leq \sigma}|F(n+\mu)| \geq\left|a_{h t}\right| \sum_{i=1}^{\sigma}\left|\frac{\Delta}{\Delta_{i, h t}}\right|
$$

But

$$
\begin{aligned}
& \frac{\Delta_{i, h t}}{\Delta}=\text { the coefficient of } z^{i-1} \text { in the polynomial } \\
& \frac{1}{\Delta} \lim _{\substack{\alpha_{k s} \rightarrow \alpha_{k} \\
\text { all ks }}}\left(\left\{\prod_{\substack{k=1 \\
k s \neq h t}}^{m} \prod_{s=1}^{\rho(k)}\left(\alpha_{k s} \frac{\partial}{\partial \alpha_{k s}}\right)^{s-1}\right\} \cdot \frac{D}{\alpha_{h t}^{n+1}} \prod_{\substack{k=1 \\
k s \neq h t}}^{m} \prod_{\substack{s=1 \\
k s t}}^{\rho(k)}\left(\frac{z-\alpha_{k s}}{\alpha_{h t}-\alpha_{k s}}\right)\right) \\
& = \pm \text { the coefficient of } z^{i-1} \text { in the polynomial }
\end{aligned}
$$


(13)

$$
\left\{\begin{array}{l}
\prod_{\substack{k=1 \\
k \neq h}}^{m}\left(\frac{z-\alpha_{k}}{\alpha_{h}-\alpha_{k}}\right)^{\rho(k)} \cdot \frac{\left(z-\alpha_{h}\right)^{t-1}}{\alpha_{h}^{n+t}(t-1) !} \times \\
\times \sum \prod_{r=t+1}^{\rho(h)} \frac{\left(\alpha_{h} \frac{\partial}{\partial \alpha_{h}}\right)^{\lambda(r)}\left\{\alpha_{h}^{n+r-\lambda(r)}(r-1-\lambda(r)) !\left(z-\alpha_{h}\right) \prod_{\substack{j=1 \\
j \neq h}}^{m}\left(\alpha_{h}-\alpha_{j}\right)^{\rho(j)}\right\}}{\alpha_{h}^{n+r}(r-1) ! \prod_{\substack{j=1 \\
j \neq h}}^{m}\left(\alpha_{h}-\alpha_{j}\right)^{\rho(j)}}
\end{array}\right.
$$

where the sum is taken over all non-negative integers $\lambda(r)$ with sum $\rho(h)-t$ such that $\lambda(\rho(h)) \geq 1$ and $\lambda(r) \leq r-t, t+1 \leq r \leq \rho(h)$.

Put

$$
\delta_{h}=\min _{\substack{1 \leq j \leq m \\ j \neq h}}\left|\alpha_{h}-\alpha_{j}\right| A=\max _{1 \leq j \leq m}\left|\alpha_{j}\right|
$$

and recall that by Lemma 2 we find the upper bound for the sum of the absolute value of the coefficients of the polynomial by finding upper bounds for the absolute value of the coefficients of products of terms of the form $\left(z-\alpha_{k}\right)$, whilst terms of the form $\left(z-\alpha_{k}\right)$ are replaced by $\left(1+\left|\alpha_{k}\right|\right)$.

We study the rather ugly sum in the expression (13), observing that

$$
\frac{1}{(t-1) !} \sum \prod_{r=t+1}^{\rho(h)} \frac{(r-1-\lambda(r)) !}{(r-1) !}<1 \text {. }
$$

Inside the product sign we have, apart from the factorials, when $\lambda(x)=1$, exactly

$$
\left(\frac{n+r-1}{\alpha_{h}}+\sum_{\substack{j=1 \\ j \neq h}}^{m} \frac{\rho_{j}}{\alpha_{h}-\alpha_{j}}\right) \quad\left(z-\alpha_{h}\right)-1
$$

which corresponds to a contribution to the upper bound of at most

$$
\frac{(n+\sigma-1)(1+A)}{\min \left(\left|\alpha_{h}\right|, \delta_{h}\right)}+1<\frac{(n+\sigma)(1+A)}{\min \left(\left|\alpha_{h}\right|, \delta_{h}\right]} \text {. }
$$

For $\lambda(r)>1$ we can then see that 


$$
\frac{(n+\sigma)^{\lambda(r)}(1+A)}{\left\{\min \left(\left|\alpha_{h}\right|, \delta_{h}\right)\right\}^{\lambda(r)}}
$$

is an appropriate contribution. Hence applying Lemma 2 to (13) we have

$$
\sum_{i=1}^{\sigma}\left|\frac{\Delta_{i, h t}}{\Delta}\right| \leq \frac{(n+\sigma)^{\rho(h)-t}(1+A)^{\sigma-1}}{\left|\alpha_{h}\right|^{n+t} \delta_{h}^{\sigma-\rho(h)}\left\{\min \left(\left|\alpha_{h}\right|, \delta_{h}\right)\right\}^{\rho(h)-t}} .
$$

Theorem 3 now follows.

\section{References}

[0] John Coates, "On the algebraic approximation of functions IV", Nederl. Akad. Wetensch. Proc. Ser. A 70 (1967), 205-212.

[1] I. Dancs, "On an extremal problem", Acta Math. Acad. Sci. Hungar. 9 (1958), 309-313.

[2] S. Dancs and P. Turán, "On the distribution of values of a class of entire functions I, II", Publ. Math. Debrecen 11 (1964), 257-272.

[3] E. Makai, "An estimation in the theory of diophantine approximations", Acta Math. Acad. Sci. Hungar. 9 (1958), 299-307.

[4] E. Makai, "The first main theorem of P. Turán", Acta Math. Acad. Sci. Hungar. 10 (1959), 405-411.

[5] E. Makai, "On a minimum problem", Ann. Univ. Sci. Budapest. Ë̈tvös Sect. Math. 3-4 (1960-61), 177-182.

[6] E. Makai, "On a minimum problem II", Acta Math. Acad. Sci. Hungar. 15 (1964), 63-66.

[7] Vera T. Sós and P. Turán, "On some new theorems in the theory of diophantine approximations", Acta Math. Acad. Sci. Hungar. 6 (1955), 241-255.

[8] Paul Turán, Eine neue Methode in der Anazysis und deren Anwendungen (Akadémiai Kiadó, Budapest, 1953). 
[9] S. Uchiyama, "A note on the second main theorem of P. Turán", Acta Math. Acad. Sci. Hungar. 9 (1958), 379-380.

University of New South Wales,

Kensinqton, New South Wales. 